\title{
PROPAGACION VEGETATIVA POR ESTACAS EN Eucalyptus camaldulensis Dehnh
}

\author{
Patricio Rojas V. (*) \\ Patricio Arce J. (**) \\ Manuel Arriagada B. (***)
}

\section{RESUMEN}

Se evalúa el arraigamiento de estacas de $\boldsymbol{E}$. camaldulensis a partir de material proveniente de rebrotes, en condiciones ambientales controladas de luz, temperatura del sustrato, temperatura ambiental, humedad relativa y riego automático.

El material colectado proviene de vástagos de árboles adultos seleccionados de las parcelas experimentales de introducción de especies de INFOR.

Se evaluó el efecto de la variabilidad individual, la concentración de hormonas y el tipo de sustrato en la capacidad de arraigamiento de estacas de $\boldsymbol{E}$. camaldulensis para bosquejar protocolos de propagación de especies del género Eucalyptus.

\begin{abstract}
This paper evaluates rooting ability of cuttings of Eucalyptus camaldulensis obtained from basal epicormic shoots, under controled conditions of light, greenhouse temperature, rooting media temperature, humidity and irrigation.

The cuttings were obtained from selected trees growing in experimental plots established as part of a species introduction program.

The effects of individual variability, hormone concentration and rooting medium on rooting capacity of $\boldsymbol{E}$. camaldulensis are evaluated, in order to determine procedures for vegetative propagation of Eucalyptus species.
\end{abstract}

(*) Ingeniero Forestal, División Silvicultura, Instituto Forestal, Huérfanos 554 - Piso 4 - Santiago - Chile.

(**) Botánico, Fisiólogo. P. Universidad Católica.

(***) Licenciado en Ingeniería Forestal, División Silvicultura, Instituto Forestal. Huérfanos 554 - Piso 4 - Santiago Chile. 


\section{INTRODUCCION}

Eucalyptus es un género de amplia distribución mundial, que se perfila como el más importante recurso maderero en la industria de la celulosa y sus derivados (DURAN CRESWELL et. al., 1985; MARTIN, 1987, TOUZET, 1980).

Las especies de este género presentan rápido crecimiento y una madera densa, por lo que son muy atractivas en el sector productivo forestal (MARTIN, 1987). Además, poseen diversos mecanismos de adaptación como yemas epicórmicas, lignotubérculos, asociaciones micorrízicas, que le permiten habitar regiones edafoclimáticas diversas (ELDRIDGE y CROMER, 1987; HARTNEY, 1980).

Es notable en las especies de Eucalyptus, su gran variabilidad intra e interespecífica en caracteres tales como: producción de biomasa, tasa de crecimiento, resistencia a heladas y déficit hídrico, entre otros (CHAPERON, 1987; ELDRIDGE y CROMER, 1987). Una forma de mantener las características favorables, evitando la variabilidad encontrada en árboles obtenidos a partir de semillas, es recurrir a la propagación vegetativa (BURDON, 1982; CAMPINHOS, 1987; CAUVIN y MARIEN, 1978; CAMPINHOS et al., 1983; MAX DE BORBA y MACHADO, 1983; POTTS y POTTS, 1986). Es por esto que la implementación de técnicas de propagación clonal en individuos seleccionados de buenas características productivas y/o resistentes a condiciones desfavorables, se presenta como una etapa crucial en programas de mejoramiento genético para futuras plantaciones comerciales.

Por esta razón el concepto de silvicultura clonal en Eucaliptos es cada vez más conocido en el mundo, desde que FRANCLET, en 1956, iniciara los trabajos de macropropagación en especies del género Eucalyptus en Marruecos (CHAPERON, 1987).

Estos clones de alta productividad reproducidos asexualmente mediante estacas (cuttings) han permitido en el programa genético iniciado por la empresa brasileña Aracruz, incrementar el rendimiento de sus bosques en un $112 \%$ (de $33 \mathrm{~m}^{3} / \mathrm{ha} /$ año a $70 \mathrm{~m}^{3} / \mathrm{ha} /$ año), mejorar las características de la pulpa (densidad, contenido) e incrementar la productividad forestal en un 135\% (de 7,85 a 18,45 toneladas de pulpa/ha/año) (GARCIA, 1984).

Actualmente millones de plantas son producidas por el sistema de estacas en el mundo (Brasil, Congo, Sudáfrica y Portugal), principalmente en especies subtropicales como Eucal. yptus grandis y marginalmente en especies de zonas templadas como Eucalyptus globulus. La técnica de propagación vegetativa se está desarrollando muy velozmente y probablemente reemplazará la producción tradicional de plantas en un futuro muy cercano (CHAPERON, 1987).

Las especies del género Eucalyptus se presentan como una atractiva alternativa de forestación en Chile, debido a su gran plasticidad, alta productividad y múltiples posibilidades de uso. Las plantaciones experimentales realizadas en el país indican que las especies de interés son: $\boldsymbol{E}$. camaldulensis, $\boldsymbol{E}$. cladocalyx, $\boldsymbol{E}$. globulus y sus subespecies maidenii y bicostata en la zona mediterránea semiárida y $\boldsymbol{E}$. regnans, $E$. nitens, $E$. delegatensis, $E$. fastigata en la zona mediterránea central (INFOR, 1986).

Entre las especies recomendadas para la zona semiárida se destaca $\boldsymbol{E}$. camaldulensis (procedencia Lake Albacutya) por su resistencia a las condiciones de sequía del secano interiot de la zona central, su buena adaptación y alta productividad (ROJAS y BARROS, 1987).

Considerando las limitaciones de las regiones semiáridas y la reconocida plasticidad que $\boldsymbol{E}$. camaldulensis posee para crecer rápidamente en sitios pobres, con poca precipitación, esta especie se presenta como buen candidato para seleccionar ecotipos para estas regiones.

Por esta razón, el objetivo de este trabajo es evaluar la técnica de propagación vegetativa por estacas, en material seleccionado de $\boldsymbol{E}$. camaldulensis, pretendiendo también bosquejarlos 
procedimientos de propagación, para un futuro programa de mejoramiento genético de este género en Chile.

\section{OBJETIVOS}

Evaluar la propagación vegetativa de $\boldsymbol{E}$. camaldulensis por medio de estacas (cuttings) provenientes de rebrotes de tocón en condiciones ambientales controladas.

Determinar el efecto de la concentración hormonal, el tipo de sustrato y el material colectado en la propagación por estacas de $\boldsymbol{E}$. camaldulensis.

Bosquejar el procedimiento de propagación por vía asexual en especies del género Eucalyptus.

\section{METODOLOGIA}

El trabajo experimental contempló el arraigamiento de estacas provenientes de rebrotes de tocón de Eucalyptus camaldulensis para diferentes tratamientos. Las variables ensayadas fueron: árbol, sustrato y concentración de Acido Indol Butírico (AIB) en el invernadero bajo condiciones ambientales controladas.

Los árboles de $\boldsymbol{E}$. camaldulensis fueron seleccionados de las parcelas experimentales del programa de introducción de especies de INFOR. Para este efecto, se incluyeron dos sitios representativos del secano costero de la V Región: Casablanca (Ensayo Santa Marta) y Peñuelas (Ensayo Reserva Forestal Peñuelas), donde la especie alcanza buen desarrollo en altura y diámetro.

\section{Obtención del Material}

Los árboles escogidos correspondieron a individuos entre 16 a 20 años, con buenas características de volumen, forma, poda natural, vigor y resistencia al ataque de hongos e insectos. El volumen de los árboles se cuantificó de acuerdo a la fórmula de OPIE (1976). La puntuación de los árboles de acuerdo al formulario de terreno permitió seleccionar mediante un programa utilitario en el computador los tres mejores individuos de ambos sitios incluidos en el estudio.

\section{CUADRO 1}

CARACTERISTICAS DE LOS ARBOLES SELECCIONADOS DE E. camaldulensis

\begin{tabular}{|l|c|c|c|}
\hline & \multicolumn{3}{|c|}{ ARBOL N } \\
\cline { 2 - 4 } & $\mathbf{1}$ & $\mathbf{2}$ & $\mathbf{3}$ \\
\hline \multirow{2}{*}{ Localidad } & Sta. Marta & Peñuelas & Penuelas \\
Parcela & 503 & 501 & 204 \\
$\mathrm{~N}^{0}$ en la parcela & 19 & 9 & 19 \\
Procedencia & Glenisla, Vic. & New South Wales, & New South Wales, \\
& Australia & Australia & Australia \\
Edad (años) & 16 & 20 & 20 \\
Altura (m) & 15,0 & 23,0 & 19.0 \\
DAP $(\mathrm{cm})$ & 23,2 & 31,0 & 26,5 \\
Vol $\left(\mathrm{m}^{3} \mathrm{scc}\right)$ & 0,2448 & 0,6343 & 0,3949 \\
\hline
\end{tabular}




\section{Corta de los Arboles}

Los árboles fueron cortados con motosierra y repasados con una sierra de arco de tal modo de dejar los tocones a una altura máxima de $12 \mathrm{~cm}$ del suelo, con un corte de bisel en $30^{\circ}$. Los tocones fueron protegidos con una malla hexagonal para evitar el daño a la rebrotación.

\section{Preparación de las Estacas}

Los vástagos obtenidos de la rebrotación de alrededor de 4 meses fueron cortados y sumergidos en una caja térmica con una solución de Benlate (fungicida sistémico) y azúcar al $5 \%$. Se seleccionaron aquellos vástagos no muy lignificados y con un largo máximo de $80 \mathrm{~cm}$.

A partir de un rebrote se obtuvieron varias estacas, desechando aquellas cuyo aspecto fuera demasiado herbáceo (ápice) o muy lignificado (base de la rama). Cada estaca debía tener 2 pares de hojas (las que se cortaron por la mitad) y un diámetro de tallo de 3 a $4 \mathrm{~mm}$.

Las estacas fueron sumergidas en una solución de $140 \mathrm{ppm}$ de Benlate durante 15 minutos. Finalmente se aplicó la hormona, introduciendo la base de la estaca $(2 \mathrm{~cm})$ en la solución de talco ácido indol butírico (AIB).

Las estacas fueron plantadas en las macetas del invernadero, de acuerdo a los tratamientos correspondientes.

\section{Diseño Estadístico}

Para evaluar la respuesta de las estacas a distintas concentraciones de hormonas, diferentes sustratos y orígenes del material colectado, se efectuó un diseño factorial al azar con tres repeticiones (Cuadro 2), en que la unidad experimental estuvo constituida por 5 estacas.

\section{CUADRO 2}

\section{DISEÑO ESTADISTICO}

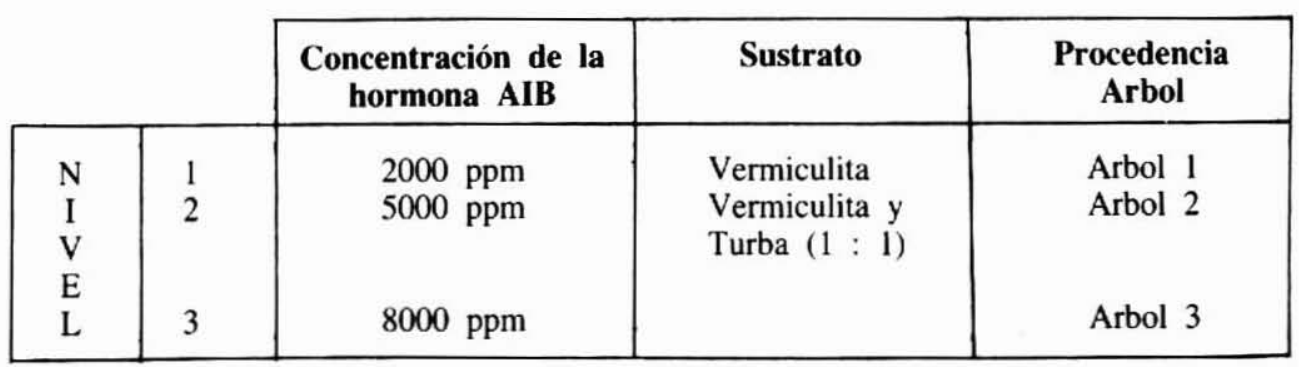

Total tratamientos: $3 \times 2 \times 3=18$

\section{Regulación de las Condiciones Ambientales}

Las macetas con las estacas fueron colocadas en el invernadero del Instituto Forestal bajo condiciones controladas de temperatura ambiental, temperatura en la base de la estaca y luminosidad, según se detalla en el Cuadro 3. Además el invernadero cuenta con un sistema de riego automático. 


\section{CUADRO 3}

CONDICIONES AMBIENTALES DE LAS ESTACAS PARA SU ARRAIGAMIENTO

\begin{tabular}{|c|c|c|c|c|c|}
\hline \multicolumn{2}{|c|}{ TEMPERATURA } & HUMEDAD & \multicolumn{2}{|c|}{ LUMINOSIDAD } & OBSERVACIONES \\
\hline $\begin{array}{c}\text { Ambiental } \\
\mathbf{0}^{\circ} \mathbf{C}\end{array}$ & $\begin{array}{c}\text { Sustrato } \\
\mathbf{0}^{\circ} \text { C }\end{array}$ & RELATIVA & $\begin{array}{c}\text { Foto }- \\
\text { Período }\end{array}$ & $\begin{array}{l}\text { Intensidad } \\
\text { Lumínica }\end{array}$ & \\
\hline $14-24$ & $16,1-25,5$ & $86 \%$ & $12 \mathrm{Hrs}$ & $\begin{array}{c}150 \\
\left(\mu \mathrm{E} / \mathrm{m}^{2} \mathrm{~s}\right)\end{array}$ & $\begin{array}{l}\text { Las estacas fueron fertilizadas } \\
\text { periódicamente con Poliverdol } \\
(1 \mathrm{gr} / \mathrm{lt}) \mathrm{y} \text { fumigadas con Benlate } \\
(140 \mathrm{ppm}) .\end{array}$ \\
\hline$\overline{\mathrm{x}}=19,3$ & $\overline{\mathrm{x}}=20,8$ & & & & \\
\hline
\end{tabular}

CUADRO 4

RESULTADOS OBTENIDOS POR TRATAMIENTO

\begin{tabular}{|c|c|c|c|c|c|}
\hline $\begin{array}{l}\text { Tratamiento } \\
\qquad(* *)\end{array}$ & $\begin{array}{l}\text { Arbol } \mathbf{N}^{\circ} \\
(* *)\end{array}$ & $\begin{array}{c}\text { Sustrato } \\
(-)\end{array}$ & $\begin{array}{l}\text { Hormona } \\
(\mathrm{ppm})(-)\end{array}$ & $\begin{array}{c}\text { Plantas con } \\
\text { Callosidades }(\%)\end{array}$ & $\begin{array}{c}\text { Plantas } \\
\text { Con raíz }(\%)\end{array}$ \\
\hline $\begin{array}{l}1 \\
2 \\
3\end{array}$ & \multirow[t]{2}{*}{1} & Vermiculita & $\begin{array}{l}2000 \\
5000 \\
8000\end{array}$ & $\begin{array}{r}46,7 \\
33,3 \\
6,7 \\
\end{array}$ & $\begin{array}{l}33,3 \\
46,7 \\
33,3 \\
\end{array}$ \\
\hline $\begin{array}{l}10 \\
11 \\
12\end{array}$ & & $\begin{array}{l}\text { Vermiculita y } \\
\text { Turba }\end{array}$ & $\begin{array}{l}2000 \\
\\
5000 \\
8000\end{array}$ & $\begin{array}{l}20,0 \\
20,0 \\
40,0\end{array}$ & $\begin{array}{l}26,7 \\
46,7 \\
26,7\end{array}$ \\
\hline $\begin{array}{l}4 \\
5 \\
6\end{array}$ & \multirow[t]{2}{*}{2} & Vermiculita & $\begin{array}{l}2000 \\
5000 \\
8000\end{array}$ & $\begin{array}{r}0,0 \\
13,3 \\
20,0\end{array}$ & $\begin{array}{l}80,0 \\
33,3 \\
20,0\end{array}$ \\
\hline $\begin{array}{l}13 \\
14 \\
15\end{array}$ & & $\begin{array}{l}\text { Vermiculita } \\
\text { y Turba }\end{array}$ & $\begin{array}{l}2000 \\
\\
5000 \\
8000 \\
\end{array}$ & $\begin{array}{l}13,3 \\
20,0 \\
20,0\end{array}$ & $\begin{array}{l}40,0 \\
60,0 \\
26,7\end{array}$ \\
\hline $\begin{array}{l}7 \\
8 \\
9\end{array}$ & \multirow[t]{2}{*}{3} & Vermiculita & $\begin{array}{l}2000 \\
5000 \\
8000\end{array}$ & $\begin{array}{r}33,3 \\
6,7 \\
13,3\end{array}$ & $\begin{array}{r}20,0 \\
13,3 \\
6,7\end{array}$ \\
\hline $\begin{array}{l}16 \\
17 \\
18\end{array}$ & & $\begin{array}{l}\text { Vermiculita } \\
\text { y Turba }\end{array}$ & $\begin{array}{l}2000 \\
5000 \\
8000\end{array}$ & $\begin{array}{r}66,7 \\
13,3 \\
6,7\end{array}$ & $\begin{array}{r}0,0 \\
20,0 \\
13,3\end{array}$ \\
\hline
\end{tabular}

$\left({ }^{*}{ }^{*}\right)$ Diferencias significativas (Alfa : 0,99 ).

(-) No significativo 


\section{RESULTADOS}

Se entregan los valores porcentuales promedios de las tres repeticiones por tratamiento según plantas que presentaron callosidades y plantas que desarrollaron sistema radicular.

De acuerdo a los resultados obtenidos del ANDEVA, existen diferencias significativas según el árbol donde se colecten las estacas (Alfa $=0,99$ ).

No existen diferencias significativas en la concentración de la hormona, ni en el sustrato utilizado, ni en las interacciones de los factores. (Alfa $=0,99$ ).

\section{DISCUSION}

Son numerosos los ejemplos de propagación vegetativa en diversas especies del género Eucalyptus (HARTNEY, 1980; CAUVIN, 1981, CELBI, 1982; CHAPERON et al., 1983; DURAN - CRESWELL et al , 1985). Muchas de tales publicaciones se refieren exclusivamente a $\boldsymbol{E}$. camaldulensis (GIORDANO, 1961; GEARY y HARDING, 1984; MONTALDI y MARCAVILLACA, 1963; BACHELARD y STOWE, 1963).

De acuerdo a los resultados obtenidos en este trabajo fue posible obtener hasta un $80 \%$ de arraigamiento en uno de los tratamientos ensayados (Fig. 1). En el resto de los tratamientos dicho porcentaje es inferior al $60 \%$, sin considerar las plantas con callosidades que pueden mejorar sustancialmente los resultados.

FIGURA 1

ARRAIGAMIENTO DE ESTACAS

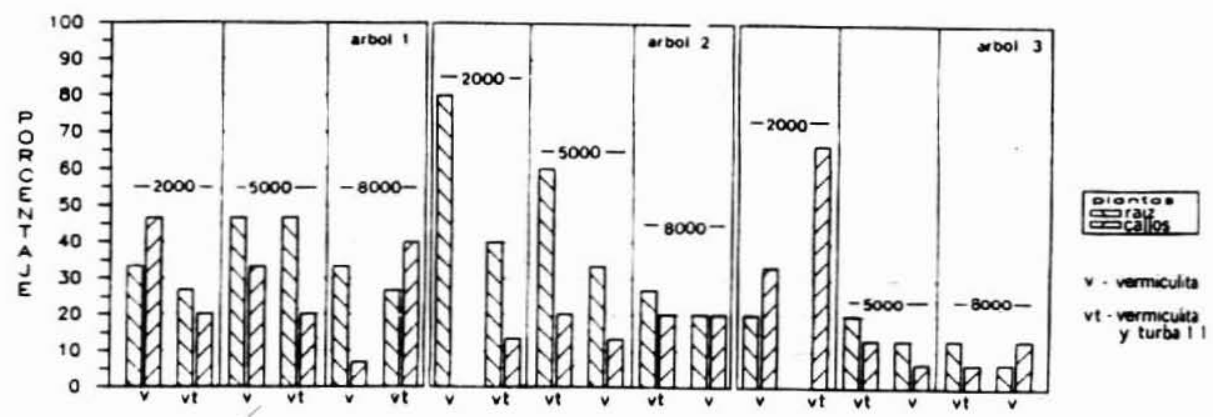

Se ha descrito que para inducir arraigamiento de estacas en numerosas especies, éstas deben ser tratadas con hormonas promotoras de raíces. BACHELARD y STOWE (1963) probaron diferentes auxinas en estacas de $\boldsymbol{E}$. camaldulensis en medio líquido y obtuvieron los mejores resultados con ácido indol butírico, en solución de $1.0 \mathrm{mg} / \mathrm{tt}$. En medio sólido, en cambio, esta hormona es comúnmente usada en diferentes especies de Eucalyptus, en concen. traciones que oscilan las 500 a 8000 ppm (CELBI, 1982; MAX DE BORBA y BRUNE, 1983: CAMPINHOS e IKEMORI, 1983).

De acuerdo a nuestros resultados, la mejor concentración hormonal fue de $2000 \mathrm{ppm}$, en la cual se obtuvo una buena formación tanto de brotes como de raíces (Fig. 2). Lo anterior está 
FIGURA 2

ARRAIGAMIENTO DE ESTACAS EN Eucalyptus camaldulensis

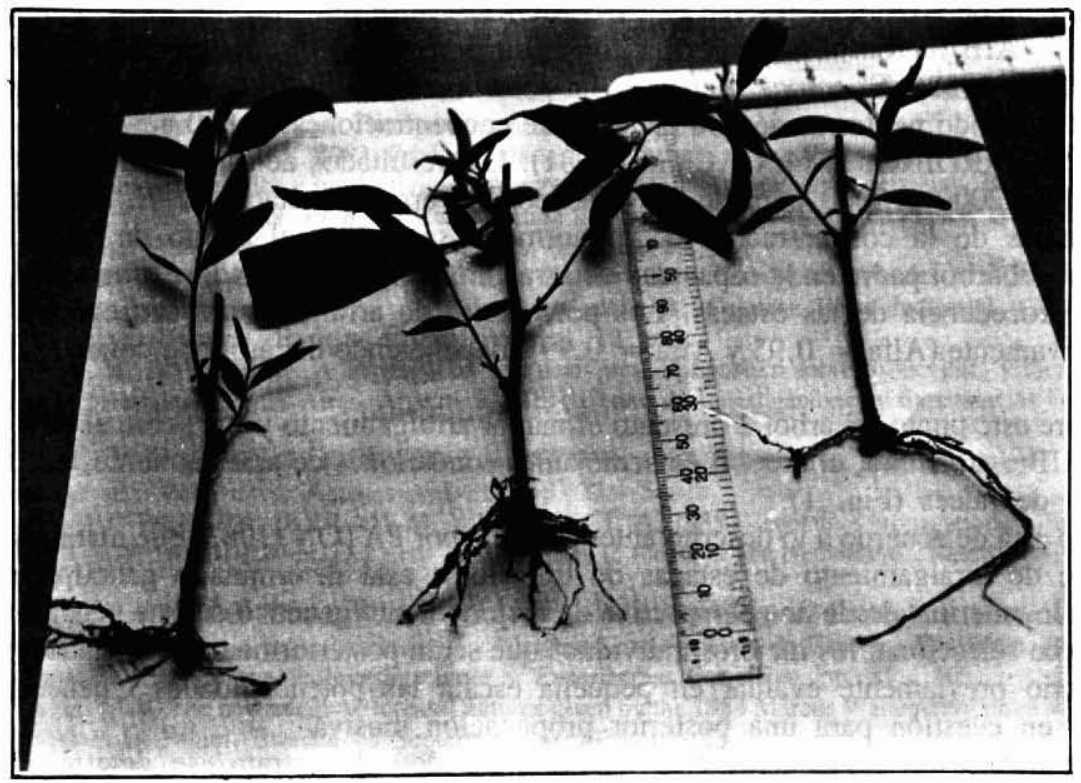

a) Estacas completas mostrando brotes y raíces.

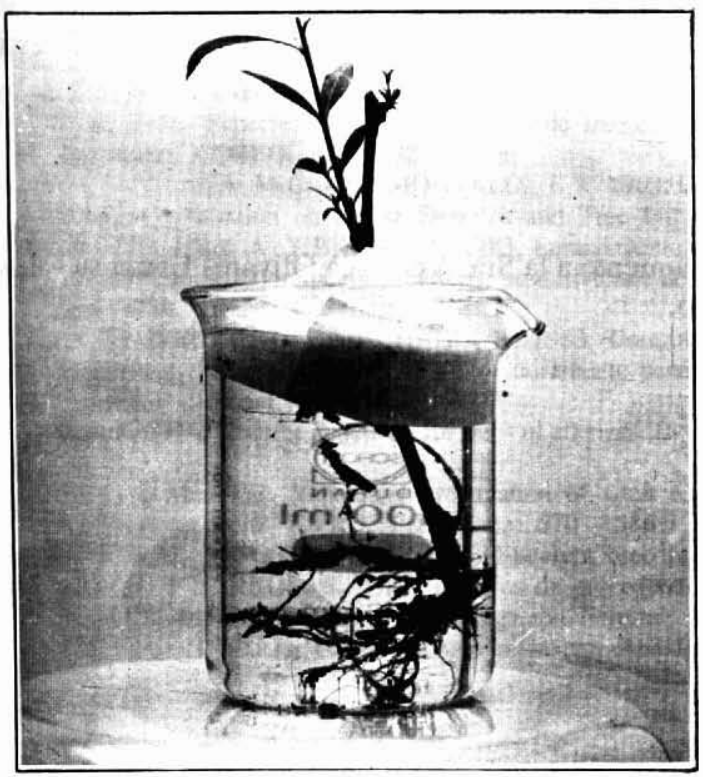

b) Detalle del vigoroso sistema radicular

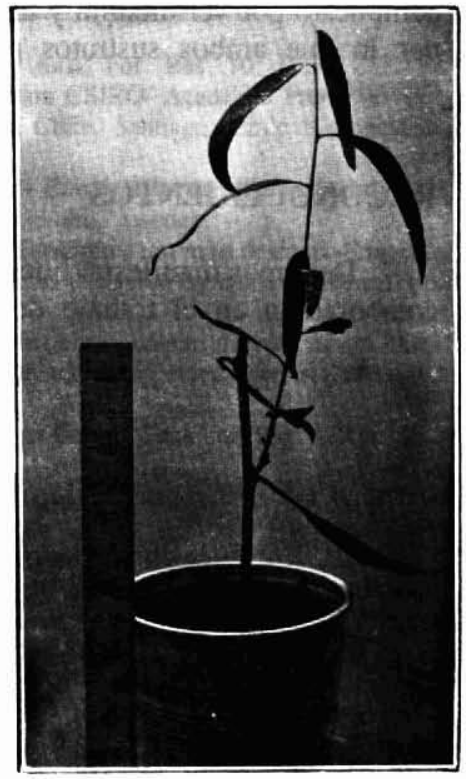

c) Plántula arraigada creciendo en macetas 
de acuerdo a lo reportado por otros autores para esta misma especie, en que han conseguido semejantes porcentajes de arraigamiento con $1600 \mathrm{ppm}$ de AIB (CAUVIN y MARIEN, 1978).

Otros autores han conseguido propagar estacas de $\boldsymbol{E}$. grandis, $\boldsymbol{E}$. saligna y $\boldsymbol{E}$. urophylla con 8000 ppm de AIB (CAMPINHOS e IKEMORI, 1983), mientras que en $\boldsymbol{E}$. globulus sólo $500 \mathrm{ppm}$ de AIB fueron necesarias para conseguir porcentajes de arraigamiento superiores al $70 \%$ (CELBI, 1982). E. camaldulensis se ha descrito como una especie muy plástica, puesto que se ha inducido neoformación de raíces entre concentraciones de 800 a 3000 ppm de AIB (GEARY y HARDING, 1984; CAUVIN, 1981). Los resultados de este estudio lo confirman, ya que entre 2000 y $8000 \mathrm{ppm}$ de AIB fue posible arraigar estacas de $\boldsymbol{E}$. camaldulensis.

Además de la concentración de la hormona se evaluó el efecto de la variabilidad individual del árbol padre en la capacidad de arraigamiento, encontrándose grandes diferencias según la procedencia de las estacas. Los porcentajes de arraigamiento conseguidos difieren significativamente $($ Alfa $=0.95$ y Alfa $=0.99)$, dependiendo del árbol de donde se colecte el material.

Sobre este punto, el árbol 2 presentó el mayor arraigamiento y fue sobre el $80 \%$ en 2000 ppm de AIB; en cambio, el árbol 3 en semejantes condiciones de arraigamiento, no consiguió inducción de raíces (Fig. 1).

Esto está de acuerdo a lo descrito anteriormente por PATON (1983) que manifiesta que el porcentaje de arraigamiento de estacas de Eucaliptos está determinado genotípicamente.

Por lo anterior, desde una perspectiva de mejoramiento genético de este género en Chile, no basta con seleccionar los mejores individuos que serán posteriormente propagados, sino que es necesario previamente evaluar en pequeña escala las potencialidades regenerativas del individuo en cuestión para una posterior propagación masiva.

Finalmente, en relación al otro factor analizado, el sustrato, se determinó que en promedio las estacas plantadas en vermiculita dieron mejores resultados que en el medio compuesto por vermiculita y turba (Cuadro 3 ), aunque las diferencias no fueron significativas, por lo que ambos sustratos pueden ser utilizados como medios de propagación de estas especies.

\section{RECONOCIMIENTOS}

Debemos manifestar nuestro reconocimiento a la Sra. Verónica Olivares L. por su valiosa colaboración en el trabajo de laboratorio. 


\section{REFERENCIAS BIBLIOGRAFICAS}

1. BACHELARD, E.P. AND STOWE, B.B. 1963. Rooting of cuttings of Acer rubrum L. and Eucalyptus camaldulensis Dehnh. Aust. J. Biol. Sci. 16: 751 - 767.

2. BURDON, R.D. 1982. The roles and optimal place of vegetative propagation in tree breeding strategies.

3. CAMPINHOS, E. 1987. Propagacao vegetativa de Eucalyptus por enraizamento de estacas. Simposio sobre Silvicultura y Mejoramiento Genético de Especies Forestales. Buenos Aires, Argentina. CIEF. Centro de Investigaciones y Experiencias Forestales. pp. 208 - 214.

4. CAMPINHOS, E. IKEMORI, Y. 1983. Producao massal de Eucalyptus spp a través de estaquia. Simposio IUFRO. En melhoramento genético e productividade de especies florestais de rapido crescimento, Brasil. pp. 770-775.

5. CAUVIN, B. Y MARIEN, J.N. 1978. La Multiplication Végétative des Eucalyptus en France. Quelques aspects de la Juvénilité et de la réjuvénilization. Annales AFOCEL pp. $141-175$.

6. CAUVIN, B. 1981. Réjuvénilization - Multiplication d'ortets séniles Eucalyptus. Annales AFOCEL pp. 74 - 105.

7. CELBI, 1982. Propagacao vegetativa do Eucalyptus globulus. Celulosa Beira Industrial (CELBI), SARL. Departamento Florestal, Figueira da Fez, Portugal. 12. p.

8. CHAPERON, H. 1987. Vegetative Propagation of Eucalyptus. Simposium sobre Silvicultura y mejoramiento de especies forestales. Buenos Aires, Argentina, CIEF, Centro de Investigaciones y Experiencias Forestales. pp. $215-232$.

9. CHAPERON. H., BERGER, M., FRAYSSE, J. et LEZE, D. 1983. Influence de la qualité du pied - mere sur le bouturage de l'Eucalyptus. Annales AFOCEL pp. 27 - 54.

10. DURAN - CRESWELL, R., BOULAY, M. Y FRANCLET, A. 1985. Vegetative propagation of Eucalyptus. In: BONGA. J.M. and DURZAN. D.J., ed. Tissue culture in forestry. The Netherlands, Martinus Nigloff pp. 150 - 181.

11. ELDRIDGE, K.G.. CROMER, R.N. 1987. Adaptation and physiology of Eucalyptus in relation to genetic improvement. Simposio sobre Silvicultura y Mejoramiento Genético de Especies Forestales. Buenos Aires, Argentina. CIEF, Centro de Investigaciones y Experiencias Forestales. pp. $85-100$.

12. GARCIA, L. 1984. The new eucalypt forest. Lectures given by the 1984 Marcus Wallemberg Prize Winners at the Symposium in Falum. Sweden on September 14, 1984. pp. 13.

13. GEARY, T. F. Y HARDING, W. G. 1984. The effects of leaf quantity and trimming on rooting success with Eucalyptus camaldulensis Dehnh. Cuttings. Commonw. for. Rev. 63 (3): 225 - 236.

14. GIORDANO. E. 1961. Mist propagation of eucalypt cuttings. In: FAO Second World Eucalypt Conference, Sao Paulo, Brasil. pp. $375-379$.

15. HARTNEY, V.J. 1980. Vegetative propagation of the Eucalyptus. In: Aust. For. Res. 10: 191 - 211.

16. HILLIS, W.E. AND BROWN, A.G. 1984. Eucalypts for wood production CSIRO/ Academic Press. 434. p.

17. INFOR. 1986. Especies Forestales Exóticas de Interés Económico para Chile. Santiago, Chile. Gerencia de Desarrollo, CORFO, AF 86/32. $167 \mathrm{p}$.

18. MARCAVILLACA, M.C. AND MONTALDI, E.R. 1963. Enraizamiento de Eucalyptus camaldulensis. En: FAO World Consultation on Forest Genetics and Tree Improvement, Stockholm, Sweden. 6. p.

19. MAX DE BORBA, A. Y BRUNE. A. 1983. Enraizamento de estacas de Eucalyptus um mito desfeito. Simposio IUFRO. Em: Melhoramento genético e produtividade de especies florestais de rapido crescimento. Brasil. pp. $758 \cdot 759$.

20. MAX DE BORBA. A. Y MACHADO, R. 1983. Controle ambiental para enraizamento de estacas em clima subtropical. Simposio IUFRO. Em: Melhoramento genético e produtividade de especies florestais de rapido crescimento, Brasil. pp. 760 - 761 .

21. OPIE, J.E. 1976. Volume functions for trees of all sizes. In: Australia. Forest Comm. Technical Paper 25 pp. 27. 30.

22. PATON, D.M. 1983. Vegetative propagation of adult eucalyptus. Colloque International sur les eucalyptus résistants au froid. IUFRO. France. pp. 570 - 586.

23. POTTS, B.M. Y POTTS, W.C. 1986. Eucalypt breeding in France. Aust. For. 49 (4): $210-218$.

24. ROJAS, P. Y BARROS, S. 1987. Ensayos de procedencias de Eucalyptus camaldulensis Dehnh en la zona semiárida de Chile. Ciencia e Investigación Forestal. 1 (1): 29 - 40.

25. TOUZET, G. 1980. La culture clonale intensive. Annales AFOCEL. pp. I-9. 